\section{P211 FEASIBILITY AND EARLY BENEFITS ACHIEVED BY ADOPTING TELEPHONE CONSULTATION AND 2-WAY REMOTE MONITORING FOR INITIATION OF CPAP THERAPY}

R Tourish, G McDowell, D MacFarlane, C Canavan, A Brown, H Ambler, C Carlin. Queen Elizabeth University Hospital, Glasgow, UK

10.1136/thoraxjnl-2017-210983.353

Introduction Obstructive sleep apnoea syndrome (OSAS) is common and UK sleep services are under considerable pressures. Adopting telephone consult based follow up supported by 2 -way remote monitoring of CPAP treatment via a cloudbased system, with usage and treatment problem alert algorithms may achieve service efficiencies, rationalise patient travel for follow up, and improve treatment quality and outcomes.

Methods In February 2017, our clinical physiology team selectively adopted 2-way remote monitoring and telephone consult follow up for initiation of auto-titrating CPAP (Airsense 10 and Airview system, ResMed) for definite or suspected OSAS, prioritising those living distant from our hospital. Data from 61 patients who had completed follow up by June 2017 was retrospectively reviewed.

Results After monitoring period and telephone consult follow up (typically requiring $15 \mathrm{~min}$ of clinician time), 44 of 61 patients were benefiting from and using CPAP therapy ongoing. Remote monitoring review triggered remote therapy adjustment in 9 patients and day-case follow up of 7 patients. 16 patients required humidifier or alternative interface posted to them. CPAP trial was concluded with no symptomatic benefit in 8 patients. 7 patients were non-compliant and non-contactable: CPAP unit return was requested. Single telephone consultation was achieved in 27 patients; 18 patients required a 2 nd call and 123 or more calls to make contact. 54 outpatient visits (median residence distance from hospital of 31 miles) were avoided, saving 3498 total travel miles.

Conclusions Adoption of 2-way remote monitoring facilitated telephone follow up of auto-CPAP initiation within a busy sleep service is feasible. Early treatment outcomes match our existing audit data for face-face follow up. Follow up hospital attendance was avoided in the majority of patients, with improvements in patient travel requirements. Remote monitoring facilitated recognition and intervention for early CPAP problems, which should in future translate into improved outcomes. Structured approach to telephone consultation and use of other communication methods should further improve service efficiency.

\section{P212 REPEATABILITY OF SELF-REPORTED SLEEPINESS IN THE CONTEXT OF FITNESS-TO-DRIVE}

${ }^{1}$ A Ayeni, 'G Beghal, ${ }^{2}$ S Steier. 'King's College London, Faculty of Life Sciences and Medicine, London, UK; '2Lane Fox Respiratory Unit, Sleep Disorders Centre, Guy's and St Thomas' NHS Foundation Trust, London, UK

\subsection{6/thoraxjnl-2017-210983.354}

Introduction Excessive daytime sleepiness (EDS) is a contributing factor to road traffic accidents, it is assessed using selfadministered questionnaires. These assessments are important information when discussing with the Driver and Vehicle Licensing Agency (DVLA) about fitness-to-drive. We hypothesised that patients may be confounded in their assessments after being informed about these potential implications. Patients and Methods This was a prospective study carried out at a tertiary hospital between June 2017 and July 2017 (registration number: 2017-7478). Patients attending clinics for sleep-disordered breathing were asked to fill in the Epworth Sleepiness Scale (ESS) and the Stanford Sleepiness Scale (SSS) prior to their clinic appointment. Following the consultation, patients were informed about the risk of EDS and driving and they were informed that the DVLA might request information based on their self-assessed sleepiness. They were then asked to fill in the questionnaires a 2nd time. Parameters recorded included age, gender, BMI and driving licence. Results of the ESS and SSS before and after clinic were compared using the student's t-test for paired observations. Subgroups of patients were analysed based on EDS (ESS $\geq 10)$ and SSS. Data are presented as mean (SD).

Results 66 subjects were included (41 males, 25 females; age 59.0 (15.7) years, BMI 32.9 (9.11), driving licence held for 27.8 (20.9) years $(n=50)$, smoking 26.9 (28.2) pack years). A total of 25 sleepy and 41 non-sleepy patients were identified. There was no difference in the ESS between the occasions [8.6 (6.2) vs $8.6(6.2)$ points; $p=0.738]$ or the SSS [2.5 (1.3) vs 2.4 (1.3) points; $p=0.255]$. Subgroup analyses based on sleepiness ESS $(p=0.108)$ and SSS $(p=0.233)$ showed no significant differences either. A total of four patients (6.1\%) changed their assessment from "sleepy" to "non-sleepy" and three patients $(4.5 \%)$ changed from "non-sleepy" to "sleepy" after receiving information about the DVLA.

Discussion Providing patients with information about the risk of driving in the context of sleepiness does not significantly change how they score the extent of their sleepiness using self-administered questionnaires, despite high intra-individual variability in about $1 / 10$ of the patients depending on the information provided.

\section{P213 IMPLEMENTATION OF A NOVEL OBSTRUCTIVE SLEEP APNOEA PATHWAY}

L Picton-Turbervill, J Butcher, S Neville, C Heaps, H Jullian, S Gunatilake. Hampshire Hospitals Foundation Trust, Basingstoke, UK

\subsection{6/thoraxjnl-2017-210983.355}

Introduction Obstructive Sleep Apnoea (OSA) is a common but under-diagnosed form of sleep disordered breathing. It causes debilitating symptoms and significant morbidity. The gold standard treatment for moderate to severe OSA is continuous positive airway pressure (CPAP) accompanied by lifestyle advice and weight loss. It is estimated that between $2 \%-4 \%$ of middle aged men and $0.5 \%-1 \%$ of women are affected and this service receives around 200 referrals per year consistent with national predictions. ${ }^{1}$ Staffing remains unchanged since service set up in 2007 with two consultant sessions and one full time nurse specialist (CNS) however due to ever increasing demand the existing service was exhibiting unacceptably long referral to treatment times (RTT).

Method 202 referrals from April 2016 to end of April 2017 were retrospectively reviewed. 101 patients were seen according to the established pathway consisting of initial clinic consultation with onward investigation and management. A novel OSA pathway was initiated in February 2017 and the next 101 consecutive patients were reviewed. All patients were 
offered a group session for respiratory polygraphy with the CNS. Patients completed a short symptom questionnaire and Epworth Sleepiness Score. These measures allowed the Consultant clinic template to increase capacity from 6 to 9 patients (50\% increase). Furthermore if CPAP is indicated set up is on the same day now as the CNS attends clinic too.

Results This new pathway improved RTT from a median of 121 days to 35 days. There was also significant improvement in diagnostic clinic outcomes from $18 \%$ to $88 \%$ (figure 1)

Conclusion Implementation of this new OSA pathway incorporating a symptom questionnaire and early diagnostic testing has enabled a significant increase in clinic capacity achieving an improvement in RTT of 70\%. Earlier treatment commencement will also lead to quicker resolution of day time somnolence limiting the time during which patients must abstain from driving and the resulting negative impact on their livelihood and lifestyle. At a time when healthcare budgets demand more for less we have demonstrated the efficacy of this new pathway.

\section{REFERENCE}

1. https://www.brit-thoracic.org.uk/document-library/clinical-information/sleep-apnoea/ impress-service-specification-for-investigation-and-treatment-of-osas/

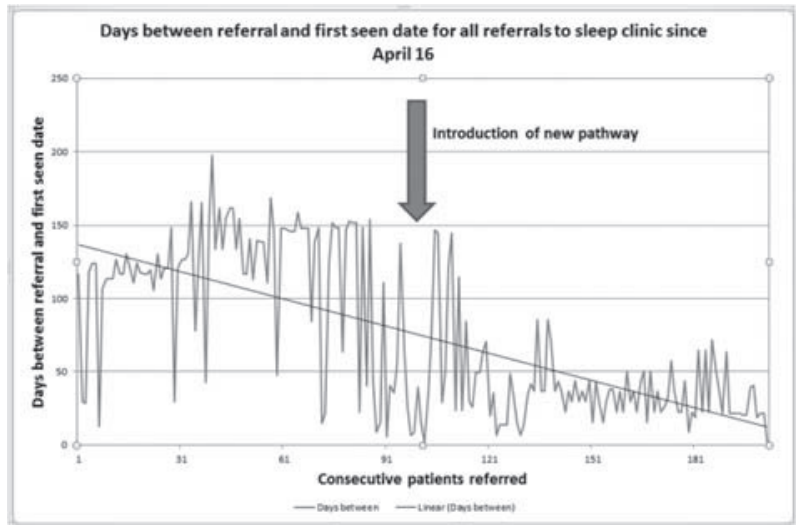

Abstract P213 Figure 1 Days between referral and first seen date for all referrals to sleep clinic since April 2016.

\section{P214 REMOTE MONITORING IN THE EARLY STAGES OF CONTINUOUS POSITIVE AIRWAY PRESSURE (CPAP) INITIATION IN OBSTRUCTIVE SLEEP APNOEA (OSA) ALLOWS EARLY DETECTION OF POOR COMPLIANCE AND MASK PROBLEMS}

S Wordingham-Baker, J O'Reilly, N Duffy, P Plant, B Chakrabarti, S Craig, A Manuel. Aintree University Hospital, Liverpool, UK

\subsection{6/thoraxjnl-2017-210983.356}

Background OSA is an increasingly recognised disease and successfully treated with CPAP. There are ever increasing demands on sleep services in the UK, in terms of provision, compliance and safety. One possible solution is the use of remote monitoring soon after CPAP set-up to determine usage patterns, residual apnoea-hypopnoea index (AHI) and mask problems. We have analysed our remote monitoring database to assess new patients with OSA set-up on CPAP.

Method Retrospective data was collected from patients with OSA commenced on CPAP from start of June 2017 for thirty days. All patients referred to the Aintree University Hospital
Sleep service had a cardio-respiratory sleep study and subsequently, if appropriate, referral for CPAP. All patients with OSA were commenced on a Resmed Airsense S10 device with humidification, using a predictive algorithm, and had an assessment for an appropriate interface. All data was collected with patient consent using Resmed Airview.

Results Data was available in 71 patients (male 70\%) for at least two weeks within this time period. Mean CPAP usage was 5 hours 4 mins with $84.5 \%$ using full face masks. Mean Residual AHI was low at 8.5 with small numbers of residual central events recorded (Table 1). See Table for more details Conclusion Remote monitoring provides a large amount of useful data which can potentially help improve CPAP provision in the UK. There is large proportion of patient with nocturnal hypoventilation, and despite effective treatment with CPAP, a group with a residual increase in AHI. Non-compliance and mask leak are identified issues and twenty patients used CPAP for less than an hour a night, with seven of those not at all. Remote monitoring allows early detection of noncompliance and an opportunity for earlier intervention to improve management in this patient group

Abstract P124 Table 1 Patient characteristics and effect of CPAP on OSA

\begin{tabular}{lll}
\hline Variable (Units) & $\mathrm{n}=71$ Mean(SD) & Range \\
\hline Age (years) & $53.2(12.4)$ & $26-80$ \\
BMI $\left(\mathrm{Kg} / \mathrm{m}^{2}\right)$ & $36.8(8.1)$ & $24-65$ \\
ESS & $13.0(5.5)$ & $2-23$ \\
Pre-treatment ODI (events/hour) & $34.5(20.0)$ & $7.1-83.4$ \\
Pre-treatment AHI (events/hour) & $36.1(23.5)$ & $5.7-104$ \\
Pre-treatment central events (events/hour) & $2.5(4.6)$ & $0-23.9$ \\
Time spent below 90\% Sp0 2 (\%) & $29.8(26.3)$ & $0-98.9$ \\
CPAP pressure (cm/ $\left.\mathrm{H}_{2} \mathrm{O}\right)$ & $9.6(1.5)$ & $6-14$ \\
Time used (hours: minutes: seconds) & $05: 04: 15(02: 49: 46)$ & $00: 00: 00-09: 33: 00$ \\
Residual AHI (events/hour) & $8.5(10.7)$ & $0-44.1$ \\
Residual Central events (events/hour) & $1.0(3.0)$ & $0-20.1$ \\
Mask leak (litres/minute) & $26.0(24.6)$ & $0-75.9$ \\
\hline
\end{tabular}

\section{P215 CONTINUOUS POSITIVE AIRWAY PRESSURE (CPAP) VERSUS AUTO-CPAP (APAP) FOR THE INITIAL TREATMENT OF OBSTRUCTIVE SLEEP APNOEA SYNDROME: CLINICAL EFFICACY AND COST}

M Mason, I Valero-Sanchez, J Archer, IE Smith. Papworth Hospital NHS Foundation Trust, Papworth Everard, Cambridge, UK

\subsection{6/thoraxjn|-2017-210983.357}

Introduction and Objectives APAP has been shown to be an effective way of titrating CPAP levels. Systematic reviews have shown similar treatment effects for APAP and CPAP. APAP devices are increasingly used for outpatient initiation of CPAP and subsequent long term use. In this service evaluation we assessed the clinical effectiveness and cost implications of APAP pressure titration followed by switching to long term CPAP.

Methods We collected data on 93, newly diagnosed patients with OSAS, starting on PAP, at baseline and at a 3 months follow-up visit. Patients initiated on APAP in an outpatient setting were asked to return in 2 weeks' time to swap APAP for CPAP, set at average pressure estimated by APAP. The 


\section{Abstract P215 Table 1}

\begin{tabular}{|c|c|c|c|}
\hline Outcome & Baseline & 3 months & P value \\
\hline ESS & $12(9-16)$ & $5(3-10)$ & 0.001 \\
\hline ODI & $23(11-45)$ & $2.9(1.7-5.4)$ & 0.001 \\
\hline Mean $\mathrm{SpO}_{2}(\%)$ & $94(92-95)$ & $96(95-97)$ & 0.001 \\
\hline BMI (kg/m $\left.{ }^{2}\right)$ & $35(30-41)$ & - & - \\
\hline Compliance (hr) & - & $5.5(3.70-6.95)$ & - \\
\hline $\begin{array}{c}\% \text { of patients using } \\
\text { CPAP } \geq 4 \text { hours }\end{array}$ & - & 74 & \\
\hline
\end{tabular}

Data are presented as median and interquartile range (IQR). A p value of $<0.05$ was deemed statiscally significant.

compliance, Epworth Sleepiness Scale (ESS) score and physiological indices; mean nocturnal $\mathrm{SpO}_{2}, 4 \%$ oxygen desaturation index (ODI), from nocturnal oximetry were collected at 3 months. Cost of device CPAP vs. long term APAP were compared. We also compared outcomes between patients with mild and moderate/severe OSA (ODI $\geq 15)$.

Results Following 3 months of treatment patients had clinically and statistically significant improvements in measured parameters aside from change in weight (Table 1). There was no significant difference in CPAP compliance between patients with mild and moderate/severe OSA, $5.6(3.2-6.9)$ vs. 5.3 (3.77.0) hours/night respectively, $p=0.9$. We found $74 \%$ of patients with mild OSA and $75 \%$ of patients with moderate/ severe OSA used CPAP for $\geq 4$ hours. Patients with mild OSA were sleepy with median ESS $12(10-15)$ and treatment with CPAP led to significant reduction to ESS 8 (4-11), at 3 months. No patients asked to be transferred back to APAP. Transferring patients to CPAP, after initial APAP pressure titration, led to a calculated cost saving of $£ 198144$ p/a, estimating that 1536 new patients initiate CPAP each year.

Conclusions - Auto CPAP titration pathway is effective in terms of the outcomes measured.

- Good compliance with significant reduction in ESS has been shown in patients with mild OSA, confirming that appropriately selected mild OSA patients can benefit from CPAP.

- Transferring patients from APAP to long term CPAP can lead to significant cost reduction.

\section{P216 ACCURACY OF SLEEP POSITION DETECTION BY SLEEP POSITIONAL TRAINERS}

S Campbell, C Carlin. Queen Elizabeth University Hospital, Glasgow, UK

\subsection{6/thoraxjnl-2017-210983.358}

Introduction Positional Obstructive Sleep Apnoea (P-OSA) where apnoeas or hypopnoeas occur predominantly or exclusively in the supine sleep position - is common. 5\% of patients attending our service for home sleep studies meet current diagnostic criteria. Sleep positional trainer (SPT) devices provide vibro-positional feedback to minimise supine sleep time. As discomfort difficulties which limited previous positional therapy approaches are potentially avoided, SPT devices may be a worthwhile alternative to CPAP therapy for P-OSA patients. Accuracy of SPT's positional analysis has however not been benchmarked. We compared positional analysis from 2 SPT devices with that from polygraphy equipment and sleep video recordings.

Methods 21 patients attending for home (Somnoscreen, SMed) or in-hospital (S-Med +video) sleep study wore additional collar (Nightshift, NS) and/or belt (Nightbalance, NB) SPT device, with vibration positional feedback disabled.\%sleep time supine for each device was determined. Concordance analysis evaluating sleep position registered by each device for 15 min epochs of sleep was performed from the NS, S-Med and video sleep positional analysis reports (this data is not reported by the Nightbalance device software).

Results Despite standard instructions, device setup failure occurred with NS device in 6 patients and NB device in 9 patients. Bland-Altman plots (figure 1) demonstrate reasonable but incomplete agreement between SPT devices, S-Med and video for\%sleep time supine. Kappa analysis demonstrated poor overall sleep positional concordance for NS vs S-Med $(\mathrm{k}=-0.002)$, NS vs video $(\mathrm{k}=0.196)$ and $\mathrm{S}$-Med vs video $(\mathrm{k}=0.092)$. Individual patient data analysis demonstrated complete sleep time concordance between NS vs S-MED or video, and S-Med vs video in some patients but consistent concordance for all 3 modalities in any individual was not seen, and there was complete discordance between modalities in other patients. Mismatch between head and torso sleep position accounted for some of these differences.

Conclusion In a real world setting we identified difficulties with SPT device setup and incomplete accuracy for sleep positional analysis by both SPT and home polygraphy equipment. Further consideration of cost-effective diagnostic precision, patient selection for SPT therapy, SPT device choice and setup and clinically relevant monitoring is required before SPT therapy is adopted. 